\title{
Effectiveness and Economic Evaluation of Therapeutic Nordic Walking in Patients with Psychosomatic Disorders: A Pragmatic Randomized Controlled Trial ${ }^{*}$
}

\author{
Dörte Watzek, Eugen \\ Mischler \\ Bern University of Applied \\ Sciences, Bern, Switzerland
}

\author{
Dorji Sonam, Barbara \\ Gubler-Blum, Claudia \\ Abbatiello \\ Bern University Hospital, \\ Bern, Switzerland
}

\author{
Lorenz Radlinger \\ Bern University of Applied \\ Sciences, Bern, Switzerland
}

\author{
Martin L. Verra \\ Bern University Hospital, \\ Bern, Switzerland
}

\begin{abstract}
The effects of exercise therapy in patients with psychosomatic disorders are modest. Therapeutic Nordic walking (tNW) might be a clinically meaningful and cost-effective additional treatment modality. This study aimed to investigate the effects of a tNW program on improving coping ability, compared to the usual care in highly disabled patients with diverse psychosomatic disorders in a hospital setting, and the willingness-to-pay for this treatment modality. Primary outcomes were self-efficacy, readiness to change health behavior, physical performance and attitude to walking. Economic evaluation was conducted with the willingness-to-pay questionnaire. Cohens' $d$ and 95\% confidence intervals were calculated for baseline, discharge and three month follow up using intention-to-treat analysis. Differences between groups were tested by $t$-test or nonparametric ANOVA. A total of 150 patients were included in this study. Results show no significant differences between the experimental and control group for any of the outcomes. Ratings of stages of change within the transtheoretical model were made by therapists or patients, they showed small effects within both groups (precontemplation: 0.2-0.4 points; preparation: 0.1-0.2; action: 0.4-1.1; maintenance: 0.4 for every comparison). There was no additional effect of the tNW program compared to the standard program in the outcome willingness-to-pay for tNW. However, since clinical experience and treatment expectancy of both patients and health care providers with this treatment modality play an important role in the treatment process, tNW might be a low-budget treatment option for some patients.
\end{abstract}

Keywords: pole walking, exercise, coping behaviors, rehabilitation, psychiatry, somatoform disorders, chronic pain

\footnotetext{
*Acknowledgements: The research team thanks all patients and physiotherapists (Julia Ratter, Bettina Gross, Agnes Oberli, Brigitte Wittwer, Daniela Benz) for their participation in the study and Joy Buchanan for her assistance with editing in English. This study was supported by the Johanna Dörmüller Foundation, Bern, Switzerland. The funder was not involved in the preparation of the study protocol, management of the trial, analysis of the data, or preparation of the manuscript. The principal investigator had full access to all the data in the study and had final responsibility for the decision to submit for publication.

Dörte Watzek, Ph.D., Health Section, Bern University of Applied Sciences.

Eugen Mischler, MBA, Health Section, Bern University of Applied Sciences.

Dorji Sonam, MSc. Physiotherapy, Department of Physiotherapy, Inselspital, Bern University Hospital.

Barbara Gubler-Blum, BSc. Physiotherapy, Department of Physiotherapy, Inselspital, Bern University Hospital.

Claudia Abbatiello, Physiotherapist, Department of Physiotherapy, Inselspital, Bern University Hospital.

Lorenz Radlinger, Ph.D., Health Section, Bern University of Applied Sciences.

Martin L. Verra, Ph.D., Department of Physiotherapy, Inselspital, Bern University Hospital.
} 


\section{Introduction}

Psychosomatic medicine is situated at the interface between psychiatric and somatic medicine (Gitlin, Levenson, \& Lyketsos, 2004). The illnesses of these patients are medically complex and generally result in high economic costs for both the individual patient and society. Chronic forms of depression, co-morbid anxiety and treatment resistance are widespread (Kruisdijk, Hendriksen, Tak, Beekman, Hopman-Rock, 2012). Forty-five percent of patients with any mental disorder meet the criteria for two or more disorders (National-Institute-of-Mental-Health (NIMH), 2013). In Switzerland, $16.9 \%$ of the population live with one and 7.7\% persons with two or more psychiatric diagnoses (Gamma \& Angst, 2001). Migrants suffer twice as often as Swiss people from depression (Altwegg et al., 2012), their language competencies are often not sufficient for cognitive therapy; therefore tNW can be seen as one possible additional modality to vary in training. Psychosomatic disorders have a serious impact on everyday life. The more diagnoses patients have, the higher the severity work or social impairment is and the worse the health-related quality of life is. Craft and Perna (2004) outlined that patients with psychosomatic or psychiatric symptoms showed a low physical activity level.

Studies about walking programs showed positive effects for different typical disorders in a psychosomatic clinic: chronic low back pain patients (Craft \& Perna, 2004; O’Connor et al., 2014) and fibromyalgia (Jones \& Liptan, 2009; Mannerkorpi, Nordeman, Cider, \& Jonsson, 2010). Another advantage of walking programs is that they are relatively low-cost interventions. The walking poles cost less than 50 Swiss francs (CHF50 is about 45 Euros or 40 US Dollars) and training sessions are generally organized in groups. For this population low costs are a requirement for a therapy that might be successfully applied in the long-term. And physical activity is non-harmful; it lacks the side-effects of medication and does not require the introspective ability necessary for most kinds of psychotherapy (Kruisdijk et al., 2012). Especially non-mother-tongue migrants benefit from the independence of lingual competencies. In a sample of primary care patients with at least two depressive episodes, about one-third of the depressed patients were successfully motivated to do regularly physical activity (Suija et al., 2009). Until now no study investigated the effects of a walking program for psychosomatic patients with multiple diagnoses.

Nordic walking is an outdoor activity. Nordic walking for patients with several medical diagnoses has to meet their needs, often they can walk only with limited speed. We call it therapeutic Nordic walking (tNW), because the range of training intensity is much wider than in normal Nordic walking and because it always includes some exercises other than walking. Therapists have a range of possibilities to adapt the training to the often low capacity of patients. Through active participation and because it is achievable for every budget it might be a valuable approach to deal with the different challenges in coping with psychosomatic diseases.

\section{Goal}

This study aimed to investigate (a) the effects of a tNW program on improving coping ability, compared to the usual care in highly disabled patients with diverse psychosomatic disorders in a hospital setting, and (b) the willingness-to-pay for this treatment modality.

\section{Methods}

\section{Trial Design}

After baseline measurement post-tests took place at discharge from the hospital and follow-up at 3-months after discharge. This randomized controlled trial is single blinded: interventions and measurements were done 
by different health professionals. Baseline and discharge assessments were done by a paper-and-pencil method and follow-up by telephone-interviews. The ethics committee in Bern (Switzerland) gave their approval on the 2009-02-26 (ref: 224/08). All participants gave written informed consent according to the Declaration of Helsinki.

\section{Setting and Participants}

The study was conducted at the Department of Psychosomatic Medicine, Bern University Hospital, Switzerland. Participants, adults with a maximum age of 65 , were inpatients in a rehabilitation program and were consecutively admitted and included in the study if they were fit enough to do walking with poles. Exclusion criteria were (a) manifest psychiatric disorder such as dementia, psychosis, suicidality; (b) psycho-intellectual inabilities; and (c) marginal knowledge of the German language (in account of questionnaires in German).

\section{Randomization and Allocation Concealment}

A non-involved occupational therapist used a computer-generated random number list to allocate patients. The principal investigator was not involved in the assessment, inclusion or treatment of the patients.

\section{Interventions}

Over the course of rehabilitation both groups took part in the standard interventions of the hospital, which consisted of the conventional multidisciplinary program including medication, nursing care, occupational, psycho- and physiotherapy.

\section{Contrast between Interventions}

The control group (CG) had no specific additional exercise interventions beside the standard rehabilitation program. A placebo intervention that would not be detected by patients as placebo was incompatible with the clinical daily routine. In addition to the therapies mentioned above, patients in the tNWG received the tNW-training, which was provided three times a week, lasting for 45 minutes. The Nordic walking classes comprised two performance levels. One level was similar to "normal" Nordic Walking, the other one was assigned to reduced tempo because of patient's low capacity. The control group was not instructed to go out regularly and did not participate at the $\mathrm{tNW}$ classes, which took place in any weather.

\section{Outcome Measures}

Socio-demographic data and medical diagnoses were obtained from the patients' medical records held at the clinic.

\section{Primary Outcome Variables}

(1) The German version of the Pain Self-Efficacy Questionnaire (PSEQ) is reliable (Cronbachs $\alpha=0.93$ ) and recommended for the measurement of changes in rehabilitation. This self-reported assessment tool measures the perceived effectiveness of patients' coping strategies, i.e., self-efficacy (Bandura, 1989). Patients have to evaluate ten items on a 6-point-Likert-scale (e.g., I can gradually be more active despite the pain/complaints). An increase of 0.3 points in patients with chronic pain was considered to be a small but significant effect (Mangels et al., 2009).

(2) A questionnaire about walking investigated attitudes towards tNW (Ratter, Benz, Oberli, \& Radlinger, 2009). The Items were "wellbeing": "When I do (Nordic) walking, I feel good"; "distraction": "(Nordic) walking takes my mind off my pain or symptoms"; "speed adaptation": "I am able to adapt the (Nordic) walking speed depending on how I feel". 
(3) For self-reports of daily activities, we used an aperture of Spinal Function Sort (SFS), which uses pictures to evaluate, if patients feel capable to do different daily activities. The pictures help patients with limited language comprehension. To estimate daily activities besides walking it seemed adequate to use only a part of this questionnaire, the goal was to avoid missing data because of our patient's limited physical condition. The relevant part was selected after expert evaluation and testing within the clinical setting. SFS has very good retest-reliability (ICC $=0.98 ; 95 \%$ CI: 0.97-1.00) (Borloz, Trippolini, Ballabeni, Luthi, \& Deriaz, 2012; Trippolini, Dijkstra, Geertzen, \& Reneman, 2015).

(4) The Freiburg Questionnaire-Stages of Chronic Pain Management (FQ-STAPM) (Maurischat et al., 2002) measures four scales based on the transtheoretical model (Prochaska \& DiClemente, 1983): pre-contemplation, preparation, action and maintenance (e.g., "I assume that I have a protracted pain problem/complaints. But there is nothing I can really change myself" stage precontemplation; "A few weeks ago I have begun to apply strategies that help me to control my pain" stage action). Internal consistency measured by Cronbachs Alpha was $0.68 \leq \alpha \leq 0.76$. Patients as well as their personal physiotherapist rated statements on these four states within the process of behavioral change.

\section{Secondary Outcome Variables}

Based on the questions on the custom-made, willingness-to-pay-questionnaire, we started by setting an anchor by asking the patients for their personal perception of the amount of 100 CHF (i.e., about 90 Euros or 90 US Dollar) because there is evidence for anchoring effects in judgment (Kahneman, 1992). Questions in the secondary outcome were (1) "You get a gift amount of 100 Swiss francs. How much of it would you spend on tNW if it makes you feel better immediately and you want to do it long-term?" (2) "Five group-sessions of tNW cost 100 CHF. Would you pay therapeutic NW yourself?" If yes: "How easy ( 1 = 'Very easy') or difficult ( 5 = 'Very difficult') would it be for you to pay this amount yourself?"

\section{Sample Size}

The sample size calculation for this study was carried out theoretically with G*Power 3.1.3 software (Faul et al., 2007) and resulted in a total sample size of $n=130$. Expecting drop-outs or violation of normal distribution, a total sample size of $n=150$ patients was appropriate for this study.

\section{Data Analysis}

Intention-to-treat analysis was carried out blinded for treatment allocation: The patients were analyzed in the treatment group to which they were randomly allocated. For missing data, imputation technique "last observation carried forward" was used. Items on the questionnaire about walking did not meet the condition of normality. So the existence of differences was examined by the global test nonparametric ANOVA (Noguchi, Gel, Brunner, \& Konietschke, 2012), for a priori planned comparisons, the Wilcoxon Rank Test was used. For other questionnaires $t$-tests were applied. For all tests, significance level 0.05 (two-tailed) was applied, for testing of the nine primary outcomes, significance level was adopted by Bonferroni-correction $(p<0.006)$. All analyses were performed using $R$, version 2.15.0.

\section{Results}

Figure 1 shows the flow of patients through the study. Patient recruitment took place between May 2009 and January 2012. A total of 706 patients with chronic musculoskeletal pain were referred to the Department of Psychosomatic Medicine, of which 150 were eligible for participation. After randomization 12 patients were 
excluded, because an exclusion criterion appeared. 138 patients were analyzed at discharge from the hospital and at a 3 month follow up. All patients but one in the CG correctly absolved no tNW training. Patients in the tNW group completed on average 7 training sessions and $2.0 \pm 0.7$ training sessions per week respectively.

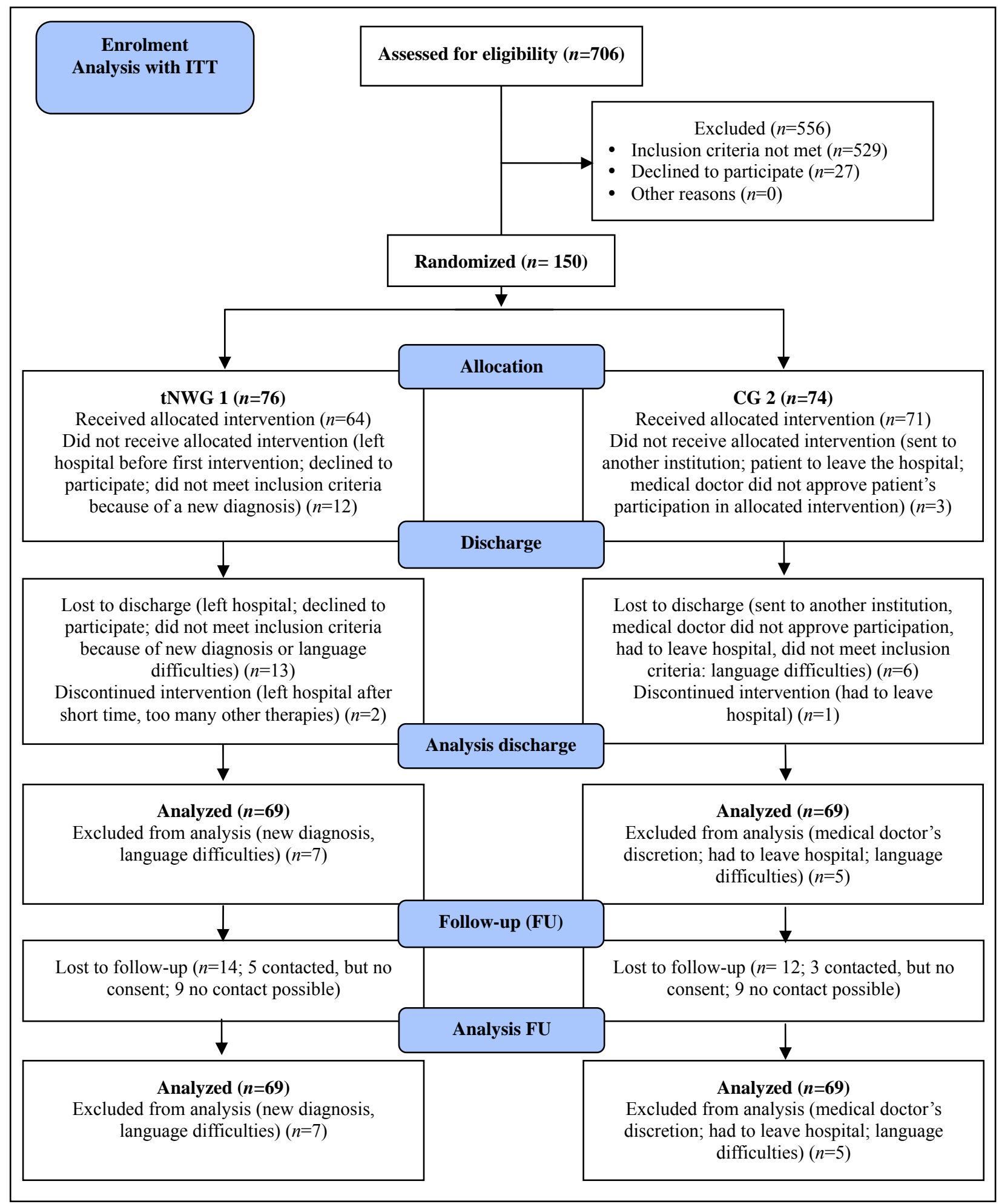

Figure 1. CONSORT diagram of flow of participants through the study. 


\section{Baseline Characteristics}

The mean age of study participants was 43.6 years (range 19-64), and $48.7 \%$ of all persons were male. Chronic pain was reported as the most frequent ICD-10 diagnosis (Dilling \& Freyberger, 2012) after depression and anxiety disorders. Baseline scores for the primary outcomes PSEQ and SFS were comparable (Table 1).

Table 1

Patient Characteristics of the Sample Groups at Baseline

\begin{tabular}{|c|c|c|}
\hline Characteristic & $\begin{array}{l}\text { Experimental group } \\
(n=69)\end{array}$ & $\begin{array}{l}\text { Control group } \\
(n=69)\end{array}$ \\
\hline \multicolumn{3}{|l|}{ Demographic characteristics } \\
\hline Male sex $(\%)$ & 46.4 & 52.2 \\
\hline Age (years; $m \pm s$ ) & $43.7 \pm 11.6$ & $43.5 \pm 10.9$ \\
\hline $\operatorname{BMI}\left(\mathrm{kg} / \mathrm{m}^{2}\right)$ & $26.3 \pm 6.3$ & $26.7 \pm 5.8$ \\
\hline \multicolumn{3}{|l|}{ Language knowledge (\%) } \\
\hline Native Swiss-German & 53.7 & 56.5 \\
\hline Good & 28.4 & 24.6 \\
\hline Average & 13.4 & 17.4 \\
\hline Minimal & 4.5 & 1.4 \\
\hline \multicolumn{3}{|l|}{ Clinical characteristics } \\
\hline \multicolumn{3}{|l|}{ Diagnosis $(\%)$} \\
\hline Chronic pain & 81.2 & 87.0 \\
\hline Depression & 65.2 & 65.2 \\
\hline Anxiety \& panic disorder & 33.3 & 27.5 \\
\hline Somatoform disorder & 20.3 & 10.1 \\
\hline Dissociative disorder & 2.9 & 2.9 \\
\hline Eating disorder & 1.4 & 1.4 \\
\hline \multicolumn{3}{|l|}{ Co morbidities (\%) } \\
\hline None & 5.8 & 1.4 \\
\hline 1 or 2 & 39.1 & 33.3 \\
\hline$>2$ & 55.1 & 65.3 \\
\hline Self-efficacy (PSEQ score; $m \pm s$ ) & $4.2 \pm 1.1$ & $4.1 \pm 1.0$ \\
\hline Functional capacity (SFS score; $\mathrm{m} \pm \mathrm{s}$ ) & $2.8 \pm 1.0$ & $2.9 \pm 1.0$ \\
\hline
\end{tabular}

\section{Primary Outcomes}

Discharge was on average after 26 days. The difference in self-efficacy between baseline and discharge from the hospital did not differ between groups (PSEQ, $d=0.19, p=0.273$ ). In both groups a small decrease in self-efficacy between baseline and discharge on a 6-point Likert-scale was observed ( $0.5 / 0.3$ points tNWG/CG).

The tNWG patients declared their own capability (SFS) at values of $2.8 / 2.9$ points (baseline/3 month follow up). In the CG the mean scores were 2.9 points for both measurements, the $t$-test showed no difference between groups $(p=0.376)$.

Results of FQ-STAPM showed that there were no significant between-group differences (patients self-rating: $p \geq 0.117$; therapist ratings: $p \geq 0.079$ ). Comparison of baseline and discharge results showed a decrease for the scale pre-contemplation and preparation in both groups (precontemplation 0.2-0.4 points; preparation: 0.1-0.2). Comparisons of the scales action and maintenance showed an increase from baseline to discharge with a difference of 0.4-1.1 points. These results apply to both therapists' rating and self-rating. 
Nonparametric ANOVA indicated no differences between groups for the items about walking attitudes (time* group effects: $p \geq 0.209$; see Table 2). Time-effects were significant for distraction and speed adaptation ( $p \leq 0.001)$, not for wellbeing $(p=0.056)$. At discharge distraction is $0.4 / 0.3$ points ( $\mathrm{tNW} / \mathrm{CG})$ better than at baseline, the scores for the 3 month follow up were comparable (difference: tNW 0.1/CG 0.2). Speed adaptation improved at discharge and did not change from discharge to the 3 month follow up. No group-effect was significant $(p \geq 0.097)$. Time and group effects are not reported in Table 2 .

Table 2

Effects of Primary Outcome Variables: Mean and Standard Deviation; Confidence Intervals for Between-Group Differences (Baseline to Discharge or Baseline to Follow Up); Between-Group Effect Sizes (Cohen's d) and p-values

\begin{tabular}{|c|c|c|c|c|}
\hline $\begin{array}{l}\text { Variable } \\
(\mathrm{m} \pm \mathrm{s})\end{array}$ & $\begin{array}{l}\text { Experimental } \\
\text { Group } \\
(n=69)\end{array}$ & $\begin{array}{l}\text { Control } \\
\text { Group } \\
(n=69) \\
\end{array}$ & $\begin{array}{l}\text { Between group difference } \\
\text { (Cohen's } d, 95 \% \mathrm{CI})\end{array}$ & $p$ \\
\hline \multicolumn{5}{|l|}{ Self-efficacy PSEQ } \\
\hline Baseline & $4.2 \pm 1.1$ & $4.1 \pm 1.0$ & & \\
\hline Discharge at 4 weeks & $3.7 \pm 1.2$ & $3.8 \pm 1.0$ & $0.19(-0.51$ to 0.14$)$ & $0.273^{1}$ \\
\hline \multicolumn{5}{|l|}{ Physical capacity SFS } \\
\hline Baseline & $2.8 \pm 1.0$ & $2.9 \pm 1.0$ & & \\
\hline Follow up at 3 months & $2.9 \pm 1.0$ & $2.9 \pm 1.0$ & $0.15(-0.11$ to 0.30$)$ & $0.376^{1}$ \\
\hline \multicolumn{5}{|c|}{ Behavioral change Patient's rating FQ-STAPM } \\
\hline \multicolumn{5}{|c|}{ Precontemplation } \\
\hline Baseline & $3.4 \pm 1.0$ & $3.5 \pm 1.0$ & & \\
\hline Discharge at 4 weeks & $3.2 \pm 1.1$ & $3.3 \pm 1.1$ & $0.01(-0.30$ to 0.31$)$ & $0.960^{1}$ \\
\hline \multicolumn{5}{|l|}{ Preparation } \\
\hline Baseline & $3.8 \pm 1.0$ & $3.8 \pm 0.8$ & & \\
\hline Discharge at 4 weeks & $3.6 \pm 0.8$ & $3.7 \pm 0.8$ & $0.10(-0.23$ to 0.42$)$ & $0.560^{1}$ \\
\hline \multicolumn{5}{|l|}{ Action } \\
\hline Baseline & $3.3 \pm 1.0$ & $3.1 \pm 1.0$ & & \\
\hline Discharge at 4 weeks & $3.7 \pm 0.9$ & $3.8 \pm 0.8$ & $0.27(-0.07$ to 0.64$)$ & $0.117^{1}$ \\
\hline \multicolumn{5}{|l|}{ Maintenance } \\
\hline Baseline & $2.1 \pm 1.0$ & $2.2 \pm 0.7$ & & \\
\hline Discharge at 4 weeks & $2.5 \pm 0.9$ & $2.6 \pm 0.8$ & $0.03(-0.28$ to 0.34$)$ & $0.854^{1}$ \\
\hline \multicolumn{5}{|c|}{ Behavioral change Therapist's rating FQ-STAPM } \\
\hline \multicolumn{5}{|c|}{ Precontemplation } \\
\hline Baseline & $3.2 \pm 0.9$ & $3.2 \pm 0.9$ & & \\
\hline Discharge at 4 weeks & $2.8 \pm 0.9$ & $2.8 \pm 1.0$ & $0.03(-0.35$ to 0.30$)$ & $0.873^{1}$ \\
\hline \multicolumn{5}{|l|}{ Preparation } \\
\hline Baseline & $3.2 \pm 0.9$ & $3.1 \pm 0.9$ & & \\
\hline Discharge at 4 weeks & $3.5 \pm 0.9$ & $3.2 \pm 0.9$ & $0.30(-0.62$ to 0.03$)$ & $0.079^{1}$ \\
\hline \multicolumn{5}{|l|}{ Action } \\
\hline Baseline & $2.3 \pm 0.9$ & $2.4 \pm 1.0$ & & \\
\hline Discharge at 4 weeks & $3.4 \pm 0.9$ & $3.3 \pm 0.8$ & $0.21(-0.59$ to 0.14$)$ & $0.226^{1}$ \\
\hline \multicolumn{5}{|l|}{ Maintenance } \\
\hline Baseline & $1.7 \pm 0.6$ & $1.7 \pm 0.6$ & & \\
\hline Discharge at 4 weeks & $2.1 \pm 0.8$ & $2.1 \pm 0.7$ & $0.07(-0.19$ to 0.30$)$ & $0.666^{1}$ \\
\hline \multicolumn{5}{|l|}{ Attitude to walking } \\
\hline \multicolumn{5}{|l|}{ Well-being } \\
\hline Baseline & $2.6 \pm 0.9$ & $2.4 \pm 1.0$ & & \\
\hline
\end{tabular}


Table 2 to be continued

\begin{tabular}{|c|c|c|c|c|}
\hline Discharge at 4 weeks & $2.3 \pm 1.2$ & $2.4 \pm 1.0$ & $0.26(-0.07$ to 0.57$)$ & \\
\hline Follow up at 3 months & $2.5 \pm 1.1$ & $2.7 \pm 1.1$ & $0.24(-0.11$ to 0.69$)$ & $0.209^{2}$ \\
\hline \multicolumn{5}{|l|}{ Distraction } \\
\hline Baseline & $3.3 \pm 1.1$ & $3.4 \pm 1.2$ & & \\
\hline Discharge at 4 weeks & $2.9 \pm 1.2$ & $3.1 \pm 1.3$ & $0.05(-0.35$ to 0.47$)$ & \\
\hline Follow up at 3 months & $3.1 \pm 1.3$ & $3.3 \pm 1.1$ & $0.06(-0.38$ to 0.56$)$ & $0.827^{2}$ \\
\hline \multicolumn{5}{|l|}{ Speed adaptation } \\
\hline Baseline & $2.7 \pm 1.4$ & $2.5 \pm 1.3$ & & \\
\hline Discharge at 4 weeks & $2.2 \pm 1.1$ & $2.1 \pm 1.1$ & $0.11(-0.30$ to 0.60$)$ & \\
\hline Follow up at 3 months & $2.2 \pm 1.3$ & $2.2 \pm 1.1$ & $0.17(-0.25$ to 0.73$)$ & $0.342^{2}$ \\
\hline
\end{tabular}

\section{Secondary Outcomes: Economic Evaluation}

Results for the statements on willingness-to-pay yielded no differences between tNWG and CG. In tNWG, 44 patients were willing to pay 100 CHF (about 90 Euros or 90 US Dollar) for five group-sessions of tNW; in CG, 50 patients said they would also pay $\left(71 \% / 75 \% ; p\left(x^{2}\right)=0.605\right)$. From a gift of $100 \mathrm{CHF}$ patients were willing to spend on average 76.7 (tNWG) or $81.1 \mathrm{CHF}(\mathrm{CG} ; p=0.507)$ for NW although it was difficult for them to pay $100 \mathrm{CHF}$ by themselves (mean score $=2.6 / 2.5 ; p=0.757$ ).

Table 3

Effects of Secondary Outcome Variables, Economic Evaluation at Discharge (Willingness-to-Pay-Questionnaire): Mean and Standard Deviation and p-values

\begin{tabular}{lccc}
\hline $\begin{array}{l}\text { Variable } \\
(\mathrm{m} \pm \mathrm{s})\end{array}$ & $\begin{array}{l}\text { Experimental } \\
\text { group } \\
(n=69)\end{array}$ & $\begin{array}{l}\text { Control } \\
\text { Group } \\
(n=69)\end{array}$ & $p$ \\
\hline${\text { Value of } 100 \mathrm{CHF}^{1}}^{\text {Willingness-to-pay }(\mathrm{CHF})^{2}}$ & $2.1 \pm 1.2$ & $1.9 \pm 1.0$ & 0.490 \\
Potentiality-to-pay $^{3}$ & $76.7 \pm 34.0$ & $81.1 \pm 29.9$ & 0.507 \\
\hline Notes. & $2.6 \pm 1.4$ & $2.5 \pm 1.3$ & 0.757 \\
\hline
\end{tabular}

Notes. $\mathrm{m}=$ mean; $\mathrm{s}=$ standard deviation; $p=$ significance level between groups for Wilcoxon rank sum test; $\mathrm{CHF}=$ Swiss Francs (100 CHF is about 90 Euros or 90 US dollar). ${ }^{1}\left(1=\right.$ "Very much", 5 = "Very little"); ${ }^{2}$ from a gift of $100 \mathrm{CHF} ;{ }^{3}$ from own money, ( 1 = "Very easy", 5 = "Very difficult").

\section{Discussion}

To the knowledge of the authors, this is the first pragmatic, randomized controlled trial evaluating the effects of additional Nordic walking in psychosomatic patients suffering from multiple diseases. An additional tNW program for patients with diverse psychosomatic disorders was not more effective than a multidisciplinary standard program alone. The willingness-to-pay also showed no differences between groups.

Several studies evaluating walking programs have shown effects for different disorders typical of patients in a psychosomatic clinic setting. The meta-analyses of O'Connor and colleagues (2014) revealed significant differences in effects of self-reported function in favor of walking interventions in adults with chronic low back pain, osteoarthritis, or fibromyalgia. 
Mead et al. (2010) found in their meta-analysis large effects for the comparison of exercise interventions with waiting list or placebo. In their study, depressive symptoms were evaluated, although patients fulfilling the full diagnosis of depression were excluded. They investigated a population less affected than the patients in the present study, who came into the clinic because of disorders resistant to outpatient therapy. In their meta-analysis Long and Van Stavel (1995) concluded that effective training programs need to last longer than 10 weeks and each training session should have duration of at least 20 minutes.

Those studies evaluating medium to high training intensities reported large or medium effect sizes (Long \& Van Stavel, 1995; Mead et al., 2010; Mannerkorpi et al., 2010). The participants in these studies were less affected by their complaints or had no or few co-morbidities (Long \& Van Stavel, 1995; Mead et al., 2010; Hartvigsen, Morsø, Bendix, \& Manniche, 2010). Patients in the present study were not able to perform higher training intensities because of fear of movement, pain or poor physical fitness. At baseline, for example, patients achieved an average walking speed of $4.5 \mathrm{~km} / \mathrm{h}$ on the treadmill, $25 \%$ of our patients stopped at a speed of $2.5 \mathrm{~km} / \mathrm{h}$, which is a very low intensity (data not reported). Therefore, in the present study tNW had to be performed at a comparatively low training intensity.

In the study by Suija and colleagues (2009) depressed patients reported that they were motivated to go walking. In accordance with these findings the patients in our study in the tNWG reported that they often felt good while walking and that they sometimes felt distracted from their pain or suffering. The drop-out rate was less than $15 \%$, which is also a sign of motivation of the participants or good adaptation of the intervention to the patients. Patients in both groups, showed a substantial willingness-to-pay for NW (CHF 79.00, on average), despite many of them being in difficult socio-economic circumstances.

Single diagnosis is rare in the field of psychosomatic medicine (NIMH, 2013; Beutel, Bleichner, von Heymann, Tritt, \& Hardt, 2010; Viniol et al., 2013). In the present study, only $3.6 \%$ of all the patients had one single diagnosis; more than $55 \%$ had more than two co-morbidities. This study was organized as part of the patient's routine within a psychosomatic clinic. This increases generalizability. Studies with less disabled patients showed bigger effects than the present study. But in the present study the focus was on patients with complicated and multiple diagnoses as frequently seen in daily practice in psychosomatic medicine.

Heterogeneity of the diagnoses increased the deviation of results and it was therefore probably more difficult to find statistically significant differences than in homogeneous samples. Mannerkorpi et al. (2010) compared two types of NW programs; they detected small effects and asked whether the contrast between the two different interventions were large enough. This is the reason why the CG in the present study had no additional supervised therapies. It is a weakness of the present study that there was limited control in clinical routine over how much stand-alone exercise was done by the CG. More stand-alone exercises in the CG might have reduced the difference between the groups. An average of two training sessions per week indicates that patients in the present study missed regularly training sessions. This might explain why the differences in effects between $\mathrm{tNWG}$ and CG were smaller than expected.

\section{Implications}

Therapeutic Nordic Walking (tNW) is a low-budget intervention. Patients with multiple diagnoses should have the possibility of gaining knowledge about this intervention and deciding whether it is an additional or alternative exercise option for them or not. In this study we did not measure a trend in change of pain experience, because the main goal of therapy in these patients was seldom a decrease in pain but rather to find 
better ways to cope with the pain. Small effects in this study dissipated after the hospital stay. It seems to be important to organize ongoing coaching to help the patients stay active at home.

\section{Future Directions}

More intensive interventions are associated with bigger effects in patients without co-morbidities. Investigation of interventions for the population of patients with more complex diseases might lead to a better understanding of patients in psychosomatic medicine. In the present study, effects were statistically non-significant at a group level, although some individuals in the tNWG did report improvement. 29 patients in tNWG increased their PSEQ scores more than the average for chronic pain patients in a validation study, in which Mangels, Schwarz, Sohr, Holme, and Rief (2009) evaluated the changes in patients with chronic pain during a rehabilitation phase. Of these 29 patients, 27 declared that Nordic walking helped them to take their minds off their pain (data not shown). A clinically relevant question for further research is to figure out which patients would benefit from this treatment. It is important to integrate the population of migrants with restricted possibly of communication due to language problems into studies in this field.

Patients who suffer from symptoms for a long time are expected to find adaptive ways of coping with their disease. Many patients did not continue tNW after their rehabilitation. Therefore, future research should explore, what are the exact needs of the patients to continue NW after a hospital stay.

\section{Conclusions}

The therapeutic Nordic walking program for highly disabled patients with several psychosomatic disorders in this study was not more effective in improving coping ability than a standard program. In our study training speed was in general very low, in studies with medium effects group worked with patients who had abilities to participate in a high intensity Nordic walking program (Mannerkorpi et al., 2010). Therapists should work with patients at a maximum of intensity, if they decide to apply a Nordic walking program. All therapists who work with migrants should consider, that for them the prevalence of depression twice as high as for Swiss people (Altwegg et al., 2012). As long as their language competencies are not sufficient for cognitive therapy, tNW can be seen as one possible variation to become active. Already in the 90s Long and Van Stavel (1995) concluded that effective training programs need to last longer than 10 weeks. If rehabilitation last only 3 weeks, it is very important to find ways for the participants to continue all activities.

\section{References}

Altwegg, D., Andreani, T., Berrut, S., Gazareth, P., Hüttner, E., Kaeser, M., Lieberherr, R., Lindner, M., Marquis, J. F., Rossel, R., Roy, E., Teotino, G., \& Wyss, N. (2012). Gesundheitsstatistik 2012. Neuchâtel: Bundesamt für Statistik (BFS).

Bandura, A. (1989). Regulation of cognitive processes through perceived self-efficacy. Dev. Psychol, 25, 729-735. doi:10.1037/0012-1649.25.5.729

Beutel, M. E., Bleichner, F., Von Heymann, F., Tritt, K., \& Hardt, J. (2010). Anxiety disorders and comorbidity in psychosomatic inpatients. Psychother Psychosom, 79, 58-58. doi:10.1159/000259419

Borloz, S., Trippolini, M. A., Ballabeni, P., Luthi, F., \& Deriaz, O. (2012). Cross-cultural adaptation, reliability, internal consistency and validation of the Spinal Function Sort (SFS) for French- and German-speaking patients with back complaints. J. Occup Rehabil, 22, 387-393. doi:10.1007/s10926-012-9356-2

Craft, L. L., \& Perna, F. M. (2004). The benefits of exercise for the clinically depressed. Prim Care Companion J. Clin Psychiatry, 6, 104. doi:10.4088/PCC.v06n0301

Dilling, H., \& Freyberger, H. J. (2012). Taschenführer zur ICD-10-Klassifikation psychischer Störungen. Bern, Huber. 
Faul, F., Erdfelder, E., Lang, A. G., \& Buchner, A. (2007). G* Power 3: A flexible statistical power analysis program for the social, behavioral, and biomedical sciences. Behav. Res. Methods, 39, 175-191. doi:10.3758/BF03193146

Gamma, A., \& Angst, J. (2001). Concurrent psychiatric comorbidity and multimorbidity in a community study: Gender differences and quality of life. Eur. Arch. Psychiatry Clin. Neurosci., 1143-1146. doi:10.1007/bf03035126

Gitlin, D. F., Levenson, J. L., \& Lyketsos, C. G. (2004). Psychosomatic medicine: A new psychiatric subspecialty. Acad. Psychiatry, 28, 4-11. doi:10.1176/appi.ap.28.1.4

Hartvigsen, J., Morsø, L., Bendix, T., \& Manniche, C. (2010). Supervised and non-supervised Nordic walking in the treatment of chronic low back pain: A single blind randomized clinical trial. BMC Musculoskelet Disord, 11, 1471-2474. doi:10.1186/1471-2474-11-30

Jones, K. D., \& Liptan, G. L. (2009). Exercise interventions in fibromyalgia: Clinical applications from the evidence. Rheum Dis. Clin. N. Am., 35, 373-391. doi:10.1016/j.rdc.2009.05.004

Kahneman, D. (1992). Reference points, anchors, norms, and mixed feelings. Organ Behav. Hum. Decis. Process, 51, $296-312$. doi:10.1016/0749-5978(92)90015-Y

Kruisdijk, F. R., Hendriksen, I. J. M., Tak, E., Beekman, A. T. F., \& Hopman-Rock, M. (2012). Effect of running therapy on depression (EFFORT-D). Design of a randomised controlled trial in adult patients (ISRCTN 1894). BMC Public Health, 12. doi:10.1186/1471-2458-12-50

Long, B. C., \& Van Stavel, R. (1995). Effects of exercise training on anxiety: A meta-analysis. J. Appl. Sport Psychol., 7, 167-189. doi:10.1080/10413209508406963

Mangels, M., Schwarz, S., Sohr, G., Holme, M., \& Rief, W. (2009). Evaluation eines deutschen Fragebogens zur Erfassung der schmerzspezifischen Selbstwirksamkeit (FESS). Diagnostica, 55, 84-93. doi:10.1026/0012-1924.55.2.84

Mannerkorpi, K., Nordeman, L., Cider, Å., \& Jonsson, G. (2010). Does moderate-to-high intensity Nordic walking improve functional capacity and pain in fibromyalgia? A prospective randomized controlled trial. Arthritis Res. Ther., $12, \mathrm{R} 189$. doi:10.1186/ar3159

Maurischat, C., Harter, M., \& Bengel, J. (2002). Der Freiburger Fragebogen—Stadien der Bewaltigung chronischer Schmerzen (FF-STABS): Faktorenstruktur, psychometrische Eigenschaften und Konstruktvalidierung. Diagnostica, 48, 190-199. doi:10.1026//0012-1924.48.4.190

Mead, G. E., Morley, W., Campbell, P., Greig, C. A., Mcmurdo, M., \& Lawlor, D. A. (2010). Exercise for depression. Cochrane Database Syst Rev, 3.

National Institute of Mental Health (NIMH). (2013). The numbers count: Mental disorders in America. Retrieved September 29, 2016 from http://www.nimh.nih.gov/health/publications/the-numbers-count-mental-disorders-in-America/index.shtml\# Mood.

Noguchi, K., Gel, Y. R., Brunner, E., \& Konietschke, F. (2012). nparLD: An R software package for the nonparametric analysis of longitudinal data in factorial experiments. J. Stat Softw, 50. doi:10.18637/jss.v050.i12

O’Connor, S. R., Tully, M. A., Ryan, B., Bleakley, C. M., Baxter, G. D., Bradley, J. M., \& Mcdonough, S. M. (2014). Walking exercise for chronic musculoskeletal pain: Systematic review and meta-analysis. Arch. Phys. Med. Rehabil., 96, 724-734. doi:10.1016/j.apmr.2014.12.003

Prochaska, J. O., \& Diclemente, C. C. (1983). Stages and processes of self-change of smoking: Toward an integrative model of change. J. Consult Clin. Psychol., 51, 390-395. doi:10.1037/0022-006X.51.3.390

Ratter, J., Benz, D., Obeli, A., \& Radlinger, L. (2009). Nordic Walking mit psychosomatischen Patienten-Ziele, Machbarkeit und Effekte. Praxis Physiotherapie, 4, 58-62.

Suija, K., Pechter, Ü., Kalda, R., Tähepõld, H., Maaroos, J., \& Maaroos, H. I. (2009). Physical activity of depressed patients and their motivation to exercise: Nordic walking in family practice. Int. J. Rehabil. Res., 32, 132-138. doi:10.1097/MRR.0b013e32831e44ef

Trippolini, M. A., Dijkstra, P. U., Geertzen, J. H. B., \& Reneman, M. F. (2015). Measurement properties of the Spinal Function Sort in patients with sub-acute whiplash-associated disorders. J. Occup. Rehabil., 1, 10. doi:10.1007/s10926-014-9559-9

Viniol, A., Jegan, N., Leonhardt, C., Brugger, M., Strauch, K., Barth, J., Baum, E., \& Becker, A. (2013). Differences between patients with chronic widespread pain and local chronic low back pain in primary care-A comparative cross-sectional analysis. BMC Musculoskelet Disord, 14, 351. doi:10.1186/1471-2474-14-351 